\title{
Combinatorics, Groups, Algorithms, and Complexity: Conference in honor of Laci Babai's 60th birthday
}

\author{
Ákos Seress ${ }^{12} \| \quad$ Mario Szegedy $\left.\right|^{3}$
}

(Guest Editors)

\author{
${ }^{1}$ School of Mathematics and Statistics, University of Western Australia, Crawley, Australia ${ }^{2}$ The Ohio State Univer- \\ sity, Dept. of Mathematics \\ ${ }^{3}$ Rutgers University, Dept. of Computer Science
}

Keywords: Combinatorics, Groups, Algorithms, and Complexity

This special issue of Discrete Mathematics \& Theoretical Computer Science is dedicated to the conference Combinatorics, Groups, Algorithms, and Complexity held at The Ohio State University between March 21-25, 2010.

Honoring László (Laci) Babai's 60th birthday, the conference explored the links between the areas mentioned in the title. These areas represent Laci's wide interests in mathematics and theoretical computer science; his work has revealed and enriched many of the interconnections between them.

We had 109 participants from North America, Europe, Asia, and Australia (31 of them from overseas), including 3 Nevanlinna prize winners, 32 students, 13 postdocs, 20 females, and 18 former and current students of Laci Babai. The program consisted of 73 talks and a problem session.

We thank all participants and speakers for the success of the conference. We wish to express our gratitude to the National Science Foundation, National Security Agency, and The Ohio State Mathematical Research Institute for their generous support.

This special issue contains papers in the conference topics, but not necessarily coinciding with the authors' talks at the conference. Each paper has been peer-reviewed. Toniann Pitassi, László Pyber, Uwe Schöning, Jiří Sgall, and Aner Shalev served with us as editors of this special issue. We thank for their work as well as for the assistance of the anonymous referees.

\footnotetext{
†Email: akosemath.ohio-state.edu.

‡Email: szegedy@cs.rutgers. edu 


\section{List of talks}

- Scott Aaronson (MIT): Arthur-Merlin and Black-Box Groups in Quantum Computing (Or, How Laci Did Quantum Stuff Without Knowing It)

- Miklós Abért (Rényi Inst., Budapest): On the Geometry of Ramanujan Graphs

- László Babai (U. Chicago): Some of my Favorite Problems

- Robert Bailey (U. Regina, Canada): Metric Dimension of Distance-regular Graphs

- Robert Beals (CCR - Princeton): Polynomial-time Theory of Matrix Groups

- Robert Burns (York U., Toronto): Transforming Normal-generating $n$-tuples of a Group into Generating $n$-tuples

- Steven Butler (U. California - Los Angeles): Shuffling with Ordered Cards

- Peter J. Cameron (Queen Mary, U. of London): Bases for Permutation Groups and Combinatorial Structures

- Sourav Chakraborty (CWI, Amsterdam): Query Complexity Lower Bounds for Reconstruction

- Paolo Codenotti (U. Chicago): Isomorphism of Hypergraphs of Low Rank in Moderately Exponential Time

- Éva Czabarka (U. South Carolina, Columbia SC): Sperner-type Problems and Results

- Alla Detinko (National U. of Ireland, Galway): Towards Efficient Computing with Matrix Groups over Infinite domains

- Oren Dinai (U. Geneva, Switzerland): Growth in Some Finite Simple Groups of Lie Type of Rank One

- John Dixon (Carleton U., Ottawa): Finite Group Representations: from Approximate to Exact

- Lance Fortnow (Northwestern U., Evanston, IL): Babai, the Wizard, and the Power of Local Interaction

- Zoltán Füredi (U. Illinois at Urbana-Champaign and Rényi Inst., Budapest): Partition-critical Hypergraphs

- Martin Fürer (Penn State U.): Combinatorial Methods for the Graph Isomorphism Problem

- Shelly Garion (Max Planck Inst., Bonn): The Product Replacement Algorithm Graph of Finite Simple Groups

- Anna Gál (U. Texas - Austin): On the Limitations of 3-query Linear Locally Decodable Codes

- Chris Godsil (U. Waterloo, Canada): Quantum Physics and Graph Spectra

- Igor Gorodezky (Cornell U.): Towards an Approximation Algorithm for the Directed All-terminal Network Reliability Problem

- Shonda Gosselin (U. Winnipeg, Canada): Paley-like Uniform Hypergraphs

- Ronald L. Graham (U. California - San Diego): Descents and Drops of a Permutation 
- Uzy Hadad (Weizmann Inst. and The Open University, Israel): On the Shortest Identity in Finite Simple Groups of Lie Type

- Péter Hajnal (U. Szeged, Hungary): On Long Alternating Non-crossing Paths in 2-equicolored Convex Sets

- Tom Hayes (U. New Mexico): Liftings of Tree-Structured Markov Chains

- Harald Helfgott (U. Bristol, U.K.): Growth of Small Sets in $\mathrm{SL}_{n}$

- Gábor Hetyei (U. North Carolina, Charlotte NC): Bernoulli Type Truncation Games and Connected Permutations

- Wilfried Imrich (U. Leoben, Austria): Products of Finite and Infinite Graphs

- Gábor Ivanyos (SzTAKI, Budapest): Modules and Maximum Rank Matrix Completion

- Delaram Kahrobaei (City U. New York): Aspects of Nonabelian Group Based Cryptography

- William M. Kantor (U. Oregon): Short Presentations of Finite Simple Groups

- Martin Kassabov (Cornell U.): Spectral Gap of Cayley Graphs of Coxeter Groups

- Subhash Khot (New York U.): Sharp Kernel Clustering Algorithms and Their Associated Grothendieck Inequalities

- Shiva Kintali (Georgia Tech, Atlanta GA): Complexity of Scarf's Lemma and Fractional Stability Problems

- Raghav Kulkarni (U. Chicago): Decision Tree Complexity, Solvable Groups, and the Distribution of Prime Numbers

- Gábor Kun (DIMACS and IAS, Princeton): Proof of the Bollobás-Catlin-Eldridge Conjecture

- Samuel Kutin (CCR - Princeton): Quantum Interpolation of Polynomials

- Gábor Lippner (Harvard U.): Parameter Testing with Borel Oracles

- Satya V. Lokam (MS Research - Bangalore): Matrix Rigidity and Complexity of Linear Transformations

- Alex Lubotzky (Hebrew U., Jerusalem): Short Presentations of Finite Simple Groups

- Eugene M. Luks (U. Oregon): Permutation Groups in Parallel: Canonical Forms

- Bojan Mohar (Simon Fraser U., Canada): Rough Structure Theorem For Symmetric Graphs with Small Separations

- Dhruv Mubayi (U. Illinois at Chicago): Coloring Simple Hypergraphs

- Jaroslav Nešetřil (Charles U., Prague): Small and Simple Representations

- Yared Nigussie (East Tennessee State U.): Finite Dualities by Forbidding a Clique Minor

- Lale Ozkahya (U. Illinois at Urbana-Champaign): On Extremal Cycle-free Subgraphs of the Hypercube 
- Péter P. Pach (Eötvös U., Budapest): On the Minimal Distance of Polynomial Near-ring Codes

- Igor Pak (U. California - Los Angeles): Generating Random Trees

- Péter P. Pálfy (Rényi Inst., Budapest): On the Isomorphism Problem of Cayley Graphs

- Gabriella Pluhár (Eötvös U., Budapest): Islands on Rectangular and Triangular Grids

- András Pongrácz (Central European U., Budapest): Compatible Functions on Permutation Groups

- Cheryl E. Praeger (U. Western Australia): Serendipity, Involutions and Regular Semisimple Matrices

- László Pyber (Rényi Inst., Budapest): Growth in Finite Simple Groups of Lie Type of Bounded Rank

- Alexander Razborov (U. Chicago): A Product Theorem in Free Groups

- Lajos Rónyai (SzTAKI, Budapest): Polynomial Functions on Finite Point Sets

- Alex Russell (U. Connecticut): Approximating the Permanent with Nonabelian Determinants

- Paul Schupp (U. Illinois at Urbana-Champaign): Cellular Automata on Cayley Graphs and Amenability

- Janos Simon (U. Chicago): Insertion Sort

- Gábor Somlai (Eötvös U., Budapest): Cayley Graphs of Elementary Abelian p-groups

- Daniel Štefankovič (U. Rochester, NY): A Graph Polynomial for Independent Sets of Bipartite Graphs

- Madhu Sudan (MIT): Invariance in Property Testing

- Zoran Sunic (Texas A\&M U.): Paired Hanoi Towers Problem

- Csaba Szabó (Eötvös U., Budapest): On the Computational Complexity of Solving Equations over Finite Groups

- Éva Tardos (Cornell U.): Price of Anarchy and Adword Auctions

- Gábor Tardos (Simon Fraser U., Canada and Rényi Inst., Budapest): An Algorithmic Proof of the Lovász Local Lemma

- György Turán (U. Illinois at Chicago and U. Szeged, Hungary): Horn Formulas or Directed Hypergraphs: Combinatorics and Complexity

- Emanuele Viola (Northeastern U., Boston): On the Complexity of Generating Distributions

- Yan Wang (Yantai U., China): Self-dual and Self-Petrie-dual Maps

- Avi Wigderson (IAS, Princeton): Groups and Expanders

- James B. Wilson (Ohio State U.): Finding Direct Products of Permutation Groups is in $P$

- Jing Xu (Capital Normal U., Beijing): Vertex transitive tournaments of order $p q$

- Şükrü Yalçınkaya (U. Western Australia): The Generalization of Siskebap Theorem and Black Box Groups 